\title{
COLOR DOPPLER EVALUATION OF THE INFLUENCE OF TYPE OF DELIVERY, SEX, POSTNATAL AGE AND TIME POST FEEDING ON FULL TERM HEALTHY NEWBORNS CEREBRAL BLOOD FLOW
}

\author{
Carlos Alberto Aranha', Henrique Manoel Lederman², Conceição A.M. Segre
}

\begin{abstract}
Objective: The purpose of this study was to evaluate with Color Doppler the influence of type of delivery, sex, postnatal age and time post feeding on full term healthy newborns cerebral blood flow. Method: 50 newborns were studied. The Doppler parameters, peak-systolic velocity, end-diastolic velocity, mean velocity, pulsatility index and resistance index, were measured in the anterior cerebral artery, middle cerebral artery, posterior cerebral artery, and basilar artery. The data were compared and analyzed by statistical tests. Informed consent was obtained from all parents, and the study was approved by institutional ethical committee and review board. Results: We observed not statistically significant differences on cerebral blood flow Doppler parameters in relation to type of delivery, sex, postnatal age and feeding in full term healthy newborns. Conclusion: We believe that the knowledge of these cerebral hemodynamic profile of newborns in the first days of life can contribute in an accurate interpretation of cranial Doppler abnormal findings when pathologic flow velocities are analyzed.
\end{abstract}

KEY WORDS: color Doppler, ultrasonography, cerebral arteries, newborn, blood flow velocity, delivery, sex postnatal age, time post feeding.

\section{Doppler colorido na avaliação da influência do tipo de parto, sexo, idade pós-natal e tempo pós-mamada} no fluxo sanguíneo cerebral em recém-nascidos a termo e saudáveis

Resumo - Objetivo: $O$ objetivo deste estudo foi avaliar com Doppler colorido a influência do tipo de parto, sexo, idade pós-natal e tempo pós-mamada no fluxo sanguíneo cerebral de recém-nascidos a termo e saudáveis. Método: Foram estudados 50 recém-nascidos. A Dopplervelocimetria foi obtida nas artérias cerebral anterior, cerebral média, cerebral posterior e basilar. Os parâmetros foram comparados e analisados pelos testes estatísticos Esta pesquisa foi aprovada pela comissão ética e de pós-graduação das instituições e o consentimento informado dos pais foi obtido em todos os casos. Resultados: Não observamos diferenças estatísticamente significativas na Dopplervelocimetria do fluxo sanguíneo cerebral em relação ao tipo de parto, sexo, idade pós-natal e tempo pós-mamada dos recém-nascidos normais e saudáveis estudados. Conclusão: Acreditamos que o conhecimento deste perfil hemodinâmico do fluxo sanguíneo cerebral de recém-nascidos nos primeiros dias de vida possa contribuir para uma acurada interpretação dos achados do Doppler cerebral quando alterações patológicas de velocidade do fluxo sanguíneo forem analisadas.

PALAVRAS CHAVE: Doppler colorido, ultra-sonografia, artérias cerebrais, recém-nascido, velocidade de fluxo sanguíneo, parto, sexo, idade, pós-natal, tempo pós-mamada.

\footnotetext{
Departamento de Diagnóstico por Imagem da Escola Paulista de Medicina da Universidade Federal de São Paulo, São Paulo SP, Brazil: 'Pós-Graduando em Radiologia Clínica e Ciências Radiológicas; ${ }^{2}$ Professor Titular; ${ }^{3}$ Livre Docente em Neonatologia pela UNIFESP, Escola Paulista de Medicina. Coordenadora do Curso de Pós-Graduação em Perinatologia do Hospital Israelita Albert Einstein, São Paulo SP, Brasil.

Received 1 December 2008, received in final form 10 February 2009. Accepted 28 April 2009.

Dr. Carlos Alberto Aranha - Departamento de Diagnóstico por Imagem da EPM / UNIFESP / Setor de Pós-Graduação - Rua Napoleão de Barros 800 / 19. Subsolo - 04024-002 São Paulo SP - Brasil. E-mail: drcarlosaranha@hotmail.com
} 
It is well known that many cerebral diseases in neonates are of circulatory origin and that fluctuations in cerebral perfusion have been recognized as a major causal factor in the pathogenesis of hemorrhagic and ischemic lesions in the preterm infant. Lou, et al. ${ }^{1}$, in their pioneering studies using the 133 Xenon clearance technique, first suggested that some critically ill infants might have a pressure-passive cerebral circulation, thus allowing abrupt changes in arterial blood pressure to be transmitted directly to the brain microvasculature, then infants with impaired regulation in the first $72 \mathrm{~h}$ of life have a significantly greater incidence of hemorrhagic and ischemic lesions.

Bada, et al. ${ }^{2}$ in 1979, was the first investigator to use Doppler ultrasonography in neonates and demonstrated changes in cerebral blood flow velocity (CBFV) and in resistance index (RI) in asphyxiated neonates, particularly in newborn infants who developed intracranial hemorrhage.

Many studies have reported the normal values of CBFV and RI in asymptomatic both preterm and term neonates during the first days of life $\mathrm{e}^{3-5}$. The CBFV in term healthy newborns increased with increasing gestational age, postnatal age and birthweight, and the RI increased significantly with increasing gestational age $\mathrm{e}^{6-8}$. However, limited information is available regarding influence of oth- er factors such as type of delivery, sex, postnatal age and feeding on CBFV, RI and pulsatitility index (PI) in neonates.

The purpose of this study was to evaluate with color Doppler the influence of type of delivery, sex, postnatal age and time post feeding on full term healthy newborns cerebral blood flow between $12 \mathrm{~h}$ and $72 \mathrm{~h}$ of life.

\section{METHOD}

This study was performed in the Department of Diagnostic Imaging of Paulista School of Medicine of Federal University of São Paulo, Brazil, as part of a thesis of postgraduate medical course by first investigator.

We evaluated 50 full term healthy neonates $(n=50)$ that were born at Hospital Albert Einstein, São Paulo, Brazil, with Apgar score 9 and 10 at 5 minutes of life, gestational age between 37 to 41 weeks (mean $38.94 \mathrm{SD} \pm 0.87$ weeks), birthweight 2.665 to 4.170 grams (mean $3.359 \mathrm{SD} \pm 385.30$ grams). 37 newborns were born by caesarean section and 13 were born vaginally (normal labor), 23 was male and 27 female, 12 with postnatal age from 12 to $24 \mathrm{~h}$ (mean 22h), 26 from 24-48h (mean 41h) and 12 from 48-72h of life (mean 66h), 17 newborns were examined th after feeding, 24 after $2 \mathrm{~h}$ and 9 after $3 \mathrm{~h}$. All infants were fed three hourly with their mothers'breast milk.

Gestational age was determined according to the mother's obstetric history, date of last menstruation and confirmed by
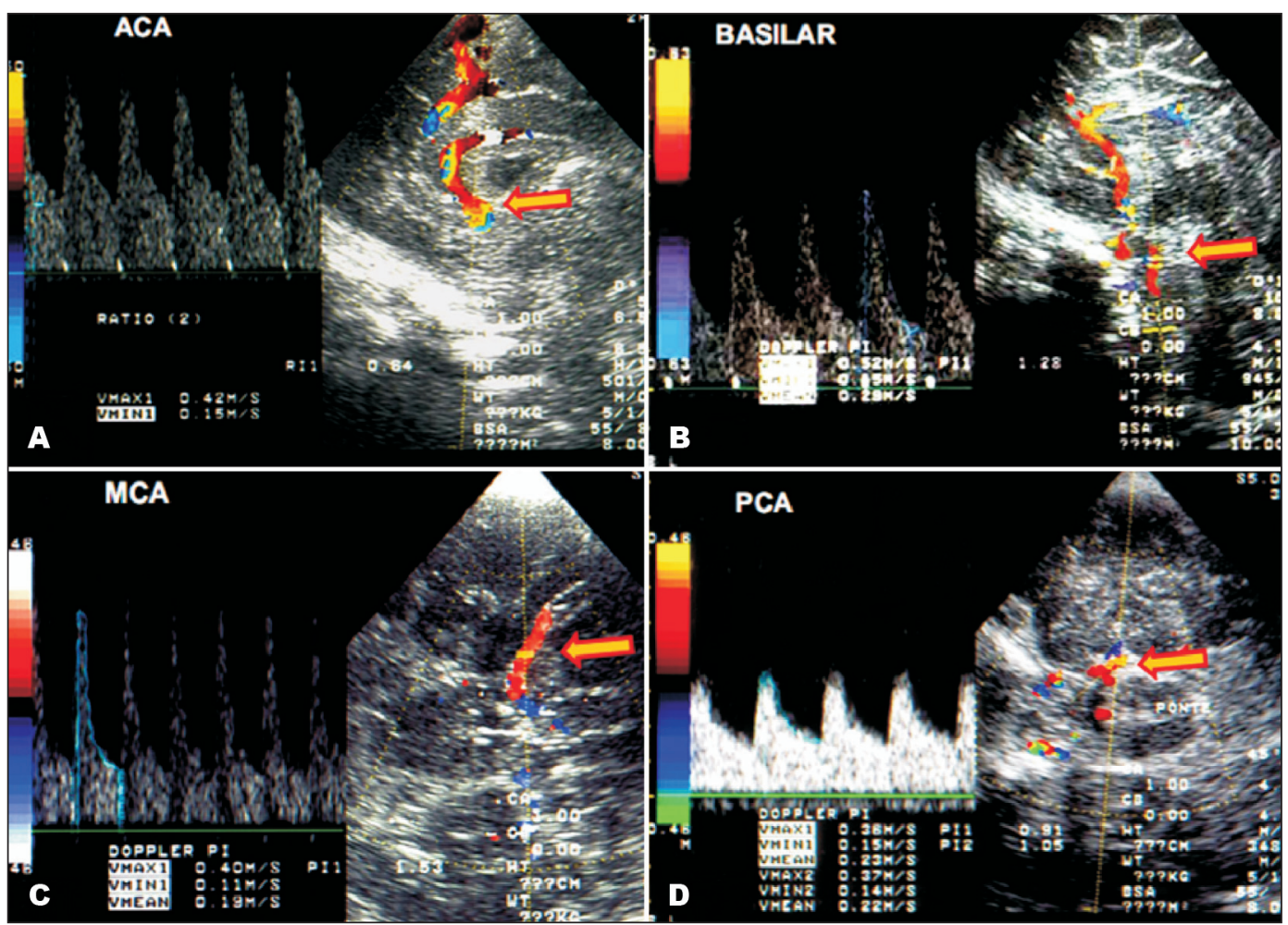

Fig 1. [A] Midline sagittal uplex Doppler scan through anterior fontanel showing on the right the color image of anterior cerebral artery (arrow) and on the left the spectral curve, and in [B] basilar artery (arrow). [C] Transaxial Duplex Doppler scan through temporal bone showing on the right the color image of M1 segment of middle cerebral artery (arrow) and on the left the spectral curve and in [D] posterior cerebral artery (arrow). 
somatic physical and neurological examination of the newborn, using the Capurro method. All infants were healthy and hemodynamically stable with normal heart rate, mean arterial blood pressure, $\mathrm{pCO} 2, \mathrm{pO} 2$ and hematocrit.

The newborns were investigated by computer sonography with color Doppler, Acuson 128 XP/10 (Mountain View, USA) and Toshiba SSH-140-A, (Nasu, Japan) with sectorial probes apropriated for neonate head. The transducer created the gray-scale image at a frequency between $5.0 \mathrm{MHz}$ to $7.0 \mathrm{MHz}$ and Doppler at a frequency at $5.0 \mathrm{MHz}$. The deep of image was set $10 \mathrm{~cm}$, sample volume was set $1 \mathrm{~mm}$ or $2 \mathrm{~mm}$, and the color sensitivity and frame rate were optimized. The high-pass filter, used to remove low frequency noise from vessel wall movement, was set at the level of 50-100 Hz. All infants were examined under criterious technical conditions in supine position when the infants was in the quiet state and eyes closed to avoid influence of ambient lighting, and with no gross body movements.

Informed consent was obtained from at least one parent, and the study was approved by both institutional ethical committee and review board.

We evaluated the right or left anterior cerebral artery (ACA), the left middle cerebral artery (LMCA), the right middle cerebral artery (RMCA), the left posterior cerebral artery (LPCA), the right posterior cerebral artery(RPCA) and the basilar artery(BA). The ACA and BA were approached through the anterior fontanel on the sagittal plane and its blood flow velocity was deter- mined at the proximal part of these vessels. The MCA and PCA arteries were approached through the temporal bone in the region above the zygomatic arch in both side. We insonated all vessels between 0 to 45 degrees and the angle correction was made when necessary (Fig 1A, B, C, D).

The CBFV measurements were obtained by cursor and automatic Fourier transform analysis from regular blood flow wave patterns over at least 4 cardiac cycles with good clear signals of spectral curve and in each artery we measured the following Doppler parameters, peak-systolic velocity (PSV), end-diastolic velocity (EDV), mean velocity, the area under the curve (MV), pulsatility index (PI) and resistance index (RI).

The RI, first described by Pourcelot ${ }^{9}$ is a blood-flow velocity waveform index, calculated by formula: RI=(PSV -EDV)/EDV, where PSV is the peak systolic velocity, EDV is the minimum forward diastolic velocity in unidirectional flow or the maximum negative velocity in diastolic flow reversal. The majority of investigators use RI as a indicative of cerebrovascular resistance because it minimizes the effect of transducer placement and can be easily obtained without velocity signal calibration ${ }^{6}$.

The pulsatility index, $\mathrm{PI}$, is an arterial blood-flow velocity waveform index designed by Gosling, et al. ${ }^{10}$, to quantify the pulsatility or oscillations of the waveform. Different definitions of this simplified PI may be found in the literature, but the following formula is commonly used: $\mathrm{PI}=(\mathrm{PSV}-\mathrm{EDV}) / \mathrm{MV}$ where PSV is the peak systolic velocity, EDV is the end diastolic velocity or

Table 1. Median, mean and SD of blood flow parameters in all arteries of our study population of 50 full term healthy newborns. Friedman teste demonstrated significant differences between the cerebral blood flow velocities, PI and RI, $p<0.001$.

\begin{tabular}{|c|c|c|c|c|c|c|c|c|c|}
\hline & & RMCA & LMCA & $\mathrm{ACA}$ & BA & RPCA & LPCA & $\mathrm{p}$ & Friedman test \\
\hline \multirow[t]{4}{*}{ PSV } & Median & 48 & 46 & 44.5 & 35.5 & 27 & 29 & $<0.001^{*}$ & $M C A(R=L)>A C A>B A>P C A(R=L)$ \\
\hline & Mean & 48.18 & 47.64 & 44.4 & 36.62 & 28.72 & 28.08 & & \\
\hline & DP & 9.09 & 10.37 & 7.42 & 7.98 & 8.25 & 7.29 & & \\
\hline & $\mathrm{n}$ & 50 & 50 & 50 & 50 & 50 & 50 & & \\
\hline \multirow[t]{4}{*}{ EDF } & Median & 14.5 & 14.5 & 16 & 12 & 11 & 10 & $<0.001^{*}$ & $\mathrm{MCA}(\mathrm{R}=\mathrm{L})=\mathrm{ACA}>\mathrm{BA}>\mathrm{PCA}(\mathrm{R}=\mathrm{L})$ \\
\hline & Mean & 15.08 & 15.14 & 15.4 & 12.66 & 10.64 & 10.46 & & \\
\hline & DP & 4.83 & 4.3 & 3.69 & 3.54 & 3.33 & 2.87 & & \\
\hline & $\mathrm{n}$ & 50 & 50 & 50 & 50 & 50 & 50 & & \\
\hline \multirow[t]{4}{*}{ MV } & Median & 26 & 27 & 26.5 & 22 & 17 & 17 & $<0.001^{*}$ & $\mathrm{MCA}(\mathrm{R}=\mathrm{L})=\mathrm{ACA}>\mathrm{BA}>\mathrm{PCA}(\mathrm{R}=\mathrm{L})$ \\
\hline & Mean & 26.48 & 26.4 & 26.14 & 21.74 & 17.74 & 17.04 & & \\
\hline & DP & 6.47 & 6.56 & 4.76 & 5.24 & 5.54 & 4.52 & & \\
\hline & $\mathrm{n}$ & 50 & 50 & 50 & 50 & 50 & 50 & & \\
\hline \multirow[t]{4}{*}{$\mathrm{PI}$} & Median & 1.24 & 1.26 & 1.09 & 1.09 & 1.05 & 1.03 & $<0.001^{*}$ & $M C A(R=L)>A C A=B A>P C A(R=L)$ \\
\hline & Mean & 1.29 & 1.25 & 1.12 & 1.12 & 1.04 & 1.04 & & \\
\hline & DP & 0.27 & 0.23 & 0.21 & 0.21 & 0.21 & 0.16 & & \\
\hline & $\mathrm{n}$ & 50 & 50 & 50 & 50 & 50 & 50 & & \\
\hline \multirow[t]{4}{*}{ RI } & Median & 0.68 & 0.68 & 0.65 & 0.65 & 0.63 & 0.63 & $<0.001^{*}$ & $\mathrm{MCA}(\mathrm{R}=\mathrm{L})>\mathrm{ACA}=\mathrm{BA}>\mathrm{PCA}(\mathrm{R}=\mathrm{L})$ \\
\hline & Mean & 0.69 & 0.68 & 0.65 & 0.65 & 0.62 & 0.62 & & \\
\hline & DP & 0.07 & 0.06 & 0.07 & 0.06 & 0.07 & 0.05 & & \\
\hline & $\mathrm{n}$ & 50 & 50 & 50 & 50 & 50 & 50 & & \\
\hline
\end{tabular}

RMCA: right middle cerebral artery; LMCA: left middle cerebral artery; ACA: anterior cerebral artery; BA: basilar artery; RPCA: right posterior cerebral artery; LPCA: left posterior cerebral artery; PSV: peak systolic velocity; EDV: end diastolic velocity; MV: mean velocity; PI: pulsatility index; RI: resistance index; $\mathrm{n}$ : number of cases. 
Table 2. Mean and medians of cerebral blood flow velocities (cm/s), PI and RI in relation to type of delivery. Mann-Whitney test ( $p>0.05)$.

\begin{tabular}{|c|c|c|c|c|c|c|}
\hline Type of delivery & & ACA-PSV & ACA-EDV & ACA-MV & ACA-PI & ACA-RI \\
\hline \multirow[t]{2}{*}{ Caesarean section $(n=37)$} & Mean & 45.59 & 15.22 & 26.57 & 1.16 & 0.66 \\
\hline & Median & 46 & 16 & 27 & 1.11 & 0.66 \\
\hline \multirow[t]{3}{*}{ Normal labor $(n=13)$} & Mean & 41 & 15.92 & 24.92 & 1.03 & 0.61 \\
\hline & Median & 41 & 16 & 24 & 1 & 0.6 \\
\hline & & RMCA-PSV & RMCA-EDV & RMCA-MV & RMCA-PI & RMCA-RI \\
\hline \multirow[t]{2}{*}{ Caesarean section $(n=37)$} & Mean & 48.97 & 15.11 & 26.57 & 1.32 & 0.69 \\
\hline & Median & 48 & 14 & 26 & 1.25 & 0.69 \\
\hline \multirow[t]{3}{*}{ Normal labor ( $n=13$ ) } & Mean & 45.92 & 15 & 26.23 & 1.21 & 0.67 \\
\hline & Median & 49 & 15 & 26 & 1.23 & 0.66 \\
\hline & & LMCA-PSV & LMCA-EDV & LMCA-MV & LMCA-PI & LMCA-RI \\
\hline \multirow{2}{*}{ Caesarean section $(n=37)$} & Mean & 48.81 & 15.08 & 26.65 & 1.28 & 0.69 \\
\hline & Median & 48 & 15 & 27 & 1.28 & 0.68 \\
\hline \multirow[t]{3}{*}{ Normal labor $(n=13)$} & Mean & 44.31 & 15.31 & 25.69 & 1.16 & 0.66 \\
\hline & Median & 44 & 14 & 27 & 1.15 & 0.66 \\
\hline & & RPCA-PSV & RPCA-EDV & RPCA-MV & RPCA-PI & RPCA-RI \\
\hline \multirow[t]{2}{*}{ Caesarean section $(n=37)$} & Mean & 29.19 & 10.84 & 18.03 & 1.03 & 0.62 \\
\hline & Median & 27 & 11 & 17 & 1.04 & 0.63 \\
\hline \multirow[t]{3}{*}{ Normal labor $(n=13)$} & Mean & 27.38 & 10.08 & 16.92 & 1.05 & 0.62 \\
\hline & Median & 27 & 11 & 18 & 1.05 & 0.63 \\
\hline & & LPCA-PSV & LPCA-EDV & LPCA-MV & LPCA-PI & LPCA-RI \\
\hline \multirow[t]{2}{*}{ Caesarean section $(n=37)$} & Mean & 28.3 & 10.32 & 17.03 & 1.05 & 0.63 \\
\hline & Median & 30 & 10 & 17 & 1.06 & 0.63 \\
\hline \multirow[t]{3}{*}{ Normal labor $(n=13)$} & Mean & 27.46 & 10.85 & 17.08 & 0.98 & 0.6 \\
\hline & Median & 27 & 10 & 16 & 0.94 & 0.6 \\
\hline & & BA-PSV & BA-EDV & BA-MV & BA-PI & BA-RI \\
\hline \multirow[t]{2}{*}{ Caesarean section ( $n=37)$} & Mean & 37.92 & 12.84 & 22.16 & 1.15 & 0.66 \\
\hline & Median & 37 & 12 & 22 & 1.14 & 0.66 \\
\hline \multirow[t]{2}{*}{ Normal labor $(n=13)$} & Mean & 32.92 & 12.15 & 20.54 & 1.04 & 0.63 \\
\hline & Median & 32 & 11 & 19 & 1 & 0.64 \\
\hline
\end{tabular}

RMCA: right middle cerebral artery; LMCA: left middle cerebral artery; ACA: anterior cerebral artery; BA: basilar artery; RPCA: right posterior cerebral artery; LPCA: left posterior cerebral artery; PSV: peak systolic velocity; EDV: end diastolic velocity; MV: mean velocity; PI: pulsatility index; RI: resistance index; n: number of cases.

minimum forward diastolic velocity in unidirectional flow or the maximum negative velocity in diastolic flow reversal, and $\mathrm{MV}$ is the area under velocity curve or velocity averaged.

\section{Statistical analysis}

The values were estimated and the relationship between these Doppler blood flow parameters and clinical factors of newborns were analyzed by statistical tests, using the software SPSS, inc. version 16.0. Data are expressed as median, mean and SD of cerebral blood flow parameters in all arteries studied. Differences in cerebral blood flow parameters between medians in the ACA, MCA, PCA and BA were assessed by Friedman test and Wilcoxon test. Comparison between medians were analyzed using Mann-Whitney test and Kruskal-Wallis test. The probability, $\mathrm{p}<0.05$ was considered statistically significant.

\section{RESULTS}

Our results demontrated that in normal clinical conditions there were differents CBFV, RI and PI between the cerebral arteries, with higher values of PSV in MCA, following by ACA, BA and PCA. Statistically significant differences were found in these parameters, showing regional differences in cerebral blood flow, that was significantly lower in the occipital region than in the frontal and parietal regions. Friedman test, $\mathrm{p}<0.001$. However, we found that there was no significant diferences in CBFV, PI and RI between the right and left MCA and also between right and left PCA, $\mathrm{p}>0.05$.

The values of CBFV, PI and RI, with medians, means and SDs of our study population of 50 full term healthy newborns are demonstrated in Table 1. 
Table 3. Mean and medians of cerebral blood flow velocities (cm/s), PI and RI in all cerebral arteries in relation to sex of newborn. MannWhitney test ( $p>0.05)$.

\begin{tabular}{|c|c|c|c|c|c|c|}
\hline Sex of newborn & & ACA-PSV & ACA-EDV & ACA-MV & ACA-PI & ACA-RI \\
\hline \multirow[t]{2}{*}{ Female $(n=27)$} & Mean & 44.93 & 15.3 & 26.22 & 1.14 & 0.65 \\
\hline & Median & 47 & 16 & 27 & 1.1 & 0.65 \\
\hline \multirow[t]{3}{*}{ Male $(n=23)$} & Mean & 43.78 & 15.52 & 26.04 & 1.1 & 0.64 \\
\hline & Median & 44 & 16 & 25 & 1.06 & 0.65 \\
\hline & & RMCA-PSV & RMCA-EDV & RMCA-MV & RMCA-PI & RMCA-RI \\
\hline \multirow[t]{2}{*}{ Female ( $n=27)$} & Mean & 50.37 & 15.07 & 27.52 & 1.31 & 0.7 \\
\hline & Median & 49 & 15 & 26 & 1.26 & 0.69 \\
\hline \multirow[t]{3}{*}{ Male $(n=23)$} & Mean & 45.61 & 15.09 & 25.26 & 1.26 & 0.67 \\
\hline & Median & 45 & 14 & 25 & 1.23 & 0.66 \\
\hline & & LMCA-PSV & LMCA-EDV & LMCA-MV & LMCA-PI & LMCA-RI \\
\hline \multirow[t]{2}{*}{ Female $(n=27)$} & Mean & 48.81 & 14.96 & 26.85 & 1.27 & 0.69 \\
\hline & Median & 47 & 15 & 27 & 1.3 & 0.68 \\
\hline \multirow[t]{3}{*}{ Male $(n=23)$} & Mean & 46.26 & 15.35 & 25.87 & 1.23 & 0.67 \\
\hline & Median & 44 & 14 & 27 & 1.22 & 0.67 \\
\hline & & RPCA-PSV & RPCA-EDV & RPCA-MV & RPCA-PI & RPCA-RI \\
\hline \multirow[t]{2}{*}{ Female $(n=27)$} & Mean & 29.04 & 10.67 & 17.93 & 1.04 & 0.63 \\
\hline & Median & 27 & 12 & 18 & 1.07 & 0.63 \\
\hline \multirow[t]{3}{*}{ Male $(n=23)$} & Mean & 28.35 & 10.61 & 17.52 & 1.04 & 0.62 \\
\hline & Median & 27 & 11 & 17 & 1 & 0.63 \\
\hline & & LPCA-PSV & LPCA-EDV & LPCA-MV & LPCA-PI & LPCA-RI \\
\hline \multirow[t]{2}{*}{ Female $(n=27)$} & Mean & 28.7 & 10.26 & 17.37 & 1.07 & 0.64 \\
\hline & Median & 30 & 10 & 18 & 1.11 & 0.64 \\
\hline \multirow[t]{3}{*}{ Male $(n=23)$} & Mean & 27.35 & 10.7 & 16.65 & 1 & 0.6 \\
\hline & Median & 28 & 10 & 16 & 1 & 0.61 \\
\hline & & BA-PSV & BA-EDV & BA-MV & BA-PI & BA-RI \\
\hline \multirow[t]{2}{*}{ Female $(n=27)$} & Mean & 36.89 & 12.67 & 22.19 & 1.11 & 0.65 \\
\hline & Median & 36 & 12 & 22 & 1.04 & 0.65 \\
\hline \multirow[t]{2}{*}{ Male $(n=23)$} & Mean & 36.3 & 12.65 & 21.22 & 1.13 & 0.65 \\
\hline & Median & 35 & 11 & 19 & 1.15 & 0.65 \\
\hline
\end{tabular}

RMCA: right middle cerebral artery; LMCA: left middle cerebral artery; ACA: anterior cerebral artery; BA: basilar artery; RPCA: right posterior cerebral artery; LPCA: left posterior cerebral artery; PSV: peak systolic velocity; EDV: end diastolic velocity; MV: mean velocity; PI: pulsatility index; RI: resistance index; n: number of cases.

When analized the CBF parameters in comparison with type of delivery, we observed not statistically significant differences. Mann-Whitney test, p>0.05 (Table 2).

When we studied the CBF parameters in comparision with sex of newborn, we observed not statistically significant differences on CBFV, PI and RI. Mann-Whitney test, p>0.05 (Table 3).

Not statistically significant differences were found in CBFV, RI and PI in relation to postnatal age of 50 full term newborns between $12 \mathrm{~h}$ and $72 \mathrm{~h}$ of life. Kruskal-Kallis test, p $>0.05$ (Table 4, Fig 2).

In relation to influence of feeding, the results also demonstrated not statistically significant differences in CBFV, RI and PI with increasing the time after feeding from 1h to 3 h. Kruskal-Kallis test, p>0.05 (Table 5, Fig 3).

\section{DISCUSSION}

Imaging modalities, as a ultrasonography, computed tomography or magnetic resonance may be normal in newborn if performed on the first day after brain anoxic injury. Neurophysiological methods, somatosensory evoked potentials, visual evoked potentials, cerebral function monitoring and near-infrared spectroscopy have proved good predictive value also during the first hours after asphyxic insult, but are not available in all centres ${ }^{11,12}$.

Color Doppler ultrasonography (CDUS) is a non-invasive imaging modality, which allows a early evaluation of newborn at bedside, repeated and safe assessment of neonatal cerebral blood flow ${ }^{13}$.

We think that is one of the first CDUS study of the influence of type of delivery, sex, postnatal age and time 
Table 4. Mean and medians of cerebral blood flow velocities $(\mathrm{cm} / \mathrm{s})$, PI and RI in all arteries studied in relation to postnatal age of newborn. Kruskal-Kallis test ( $p>0.05)$.

\begin{tabular}{|c|c|c|c|c|c|c|c|c|}
\hline Postnatal age (hours) & & & $\mathrm{n}$ & ACA-PSV & ACA-EDV & ACA-MV & ACA-PI & ACA-RI \\
\hline \multirow[t]{2}{*}{$12<24 h$} & Mean & $22 \mathrm{~h}$ & 12 & 46.25 & 16.17 & 27.58 & 1.1 & 0.64 \\
\hline & Median & $24 \mathrm{~h}$ & & 44.5 & 16 & 26 & 1.1 & 0.67 \\
\hline \multirow[t]{2}{*}{$24<48 h$} & Mean & $41 \mathrm{~h}$ & 26 & 42.73 & 14.96 & 25.23 & 1.12 & 0.65 \\
\hline & Median & $48 \mathrm{~h}$ & & 43.5 & 16 & 24.5 & 1.05 & 0.64 \\
\hline \multirow{3}{*}{$48<72 h$} & Mean & $66 \mathrm{~h}$ & 12 & 46.17 & 15.58 & 26.67 & 1.16 & 0.66 \\
\hline & Median & $72 \mathrm{~h}$ & & 48 & 14.5 & 17 & 1.16 & 0.68 \\
\hline & & & & RMCA-PSV & RMCA-EDV & RMCA-MV & RMCA-PI & RMCA-RI \\
\hline \multirow[t]{2}{*}{$12<24 h$} & Mean & $22 \mathrm{~h}$ & 12 & 50 & 15 & 27.5 & 1.32 & 0.7 \\
\hline & Median & $24 \mathrm{~h}$ & & 47 & 14.5 & 27.5 & 1.3 & 0.72 \\
\hline \multirow[t]{2}{*}{$24<48 h$} & Mean & $41 \mathrm{~h}$ & 26 & 47.69 & 15.27 & 26.73 & 1.24 & 0.68 \\
\hline & Median & $48 \mathrm{~h}$ & & 47.5 & 15 & 26 & 1.22 & 0.66 \\
\hline \multirow[t]{3}{*}{$48<72 h$} & Mean & $66 \mathrm{~h}$ & 12 & 47.42 & 14.75 & 24.92 & 1.37 & 0.69 \\
\hline & Median & $72 \mathrm{~h}$ & & 50 & 14 & 25 & 1.34 & 0.69 \\
\hline & & & & LMCA-PSV & LMCA-EDV & LMCA-MV & LMCA-PI & LMCA-RI \\
\hline \multirow[t]{2}{*}{$12<24 h$} & Mean & $22 \mathrm{~h}$ & 12 & 46.92 & 14.33 & 25.25 & 1.31 & 0.69 \\
\hline & Median & $24 \mathrm{~h}$ & & 46 & 15.5 & 27 & 1.3 & 0.68 \\
\hline \multirow[t]{2}{*}{$24<48 h$} & Mean & $41 \mathrm{~h}$ & 26 & 45.81 & 14.42 & 25.5 & 1.25 & 0.68 \\
\hline & Median & $48 \mathrm{~h}$ & & 44.5 & 14 & 25.5 & 1.23 & 0.68 \\
\hline \multirow[t]{3}{*}{$48<72 h$} & Mean & $66 \mathrm{~h}$ & 12 & 52.33 & 17.5 & 29.5 & 1.2 & 0.66 \\
\hline & Median & $72 \mathrm{~h}$ & & 57 & 16.5 & 30.5 & 1.21 & 0.65 \\
\hline & & & & RPCA-PSV & RPCA-EDV & RPCA-MV & RPCA-PI & RPCA-RI \\
\hline \multirow[t]{2}{*}{$12<24 h$} & Mean & $22 \mathrm{~h}$ & 12 & 30.92 & 10.58 & 18.42 & 1.13 & 0.66 \\
\hline & Median & $24 \mathrm{~h}$ & & 31 & 11 & 17 & 1.13 & 0.66 \\
\hline \multirow[t]{2}{*}{$24<48 h$} & Mean & $41 \mathrm{~h}$ & 26 & 27.12 & 10.23 & 16.81 & 1.02 & 0.62 \\
\hline & Median & $48 \mathrm{~h}$ & & 26 & 11 & 17 & 1.02 & 0.63 \\
\hline \multirow[t]{3}{*}{$48<72 h$} & Mean & $66 \mathrm{~h}$ & 12 & 30 & 11.58 & 19.08 & 0.99 & 0.61 \\
\hline & Median & $72 \mathrm{~h}$ & & 27.5 & 11 & 17 & 0.98 & 0.61 \\
\hline & & & & LPCA-PSV & LPCA-EDV & LPCA-MV & LPCA-PI & LPCA-RI \\
\hline \multirow[t]{2}{*}{$12<24 h$} & Mean & $22 \mathrm{~h}$ & 12 & 27 & 9.83 & 16.17 & 1.05 & 0.62 \\
\hline & Median & $24 \mathrm{~h}$ & & 30 & 10 & 17.5 & 1.11 & 0.64 \\
\hline \multirow[t]{2}{*}{$24<48 h$} & Mean & $41 \mathrm{~h}$ & 26 & 29.15 & 10.85 & 17.77 & 1.04 & 0.62 \\
\hline & Median & $48 \mathrm{~h}$ & & 29 & 10 & 17 & 1.06 & 0.63 \\
\hline \multirow[t]{3}{*}{$48<72 h$} & Mean & $66 \mathrm{~h}$ & 12 & 26.83 & 10.25 & 16.33 & 1.02 & 0.62 \\
\hline & Median & $72 \mathrm{~h}$ & & 25 & 9.5 & 15.5 & 1 & 0.61 \\
\hline & & & & BA-PSV & BA-EDV & BA-MV & BA-PI & BA-RI \\
\hline \multirow[t]{2}{*}{$12<24 h$} & Mean & $22 \mathrm{~h}$ & 12 & 38.92 & 12.83 & 22.92 & 1.15 & 0.66 \\
\hline & Median & $24 \mathrm{~h}$ & & 37.5 & 11.5 & 22.5 & 1.15 & 0.67 \\
\hline \multirow[t]{2}{*}{$24<48 h$} & Mean & $41 \mathrm{~h}$ & 26 & 35.42 & 12.62 & 21.38 & 1.08 & 0.64 \\
\hline & Median & $48 \mathrm{~h}$ & & 34 & 12 & 22 & 1.02 & 0.65 \\
\hline \multirow[t]{2}{*}{$48<72 h$} & Mean & $66 \mathrm{~h}$ & 12 & 36.92 & 12.58 & 21.33 & 1.19 & 0.66 \\
\hline & Median & $72 \mathrm{~h}$ & & 35.5 & 12 & 20 & 1.13 & 0.65 \\
\hline
\end{tabular}

RMCA: right middle cerebral artery; LMCA: left middle cerebral artery; ACA: anterior cerebral artery; BA: basilar artery; RPCA: right posterior cerebral artery; LPCA: left posterior cerebral artery; PSV: peak systolic velocity; EDV: end diastolic velocity; MV: mean velocity; PI: pulsatility index; RI: resistance index; $n$ : number of cases.

post feeding on full term healthy newborns cerebral blood flow.

Hansen et al..$^{14}$ demonstrated a linear correlation between cerebral blood flow (CBF) and area under the velocity curve (mean velocity, MV) measured by radionu- clide-labeled microspheres in piglets, and that Doppler blood flow velocity could be used to provide a noninvasive estimate of $\mathrm{CBF}$ in neonates. Other experimental research in lambs have demonstrated that Doppler blood flow estimates and mean blood flow velocities correlate 

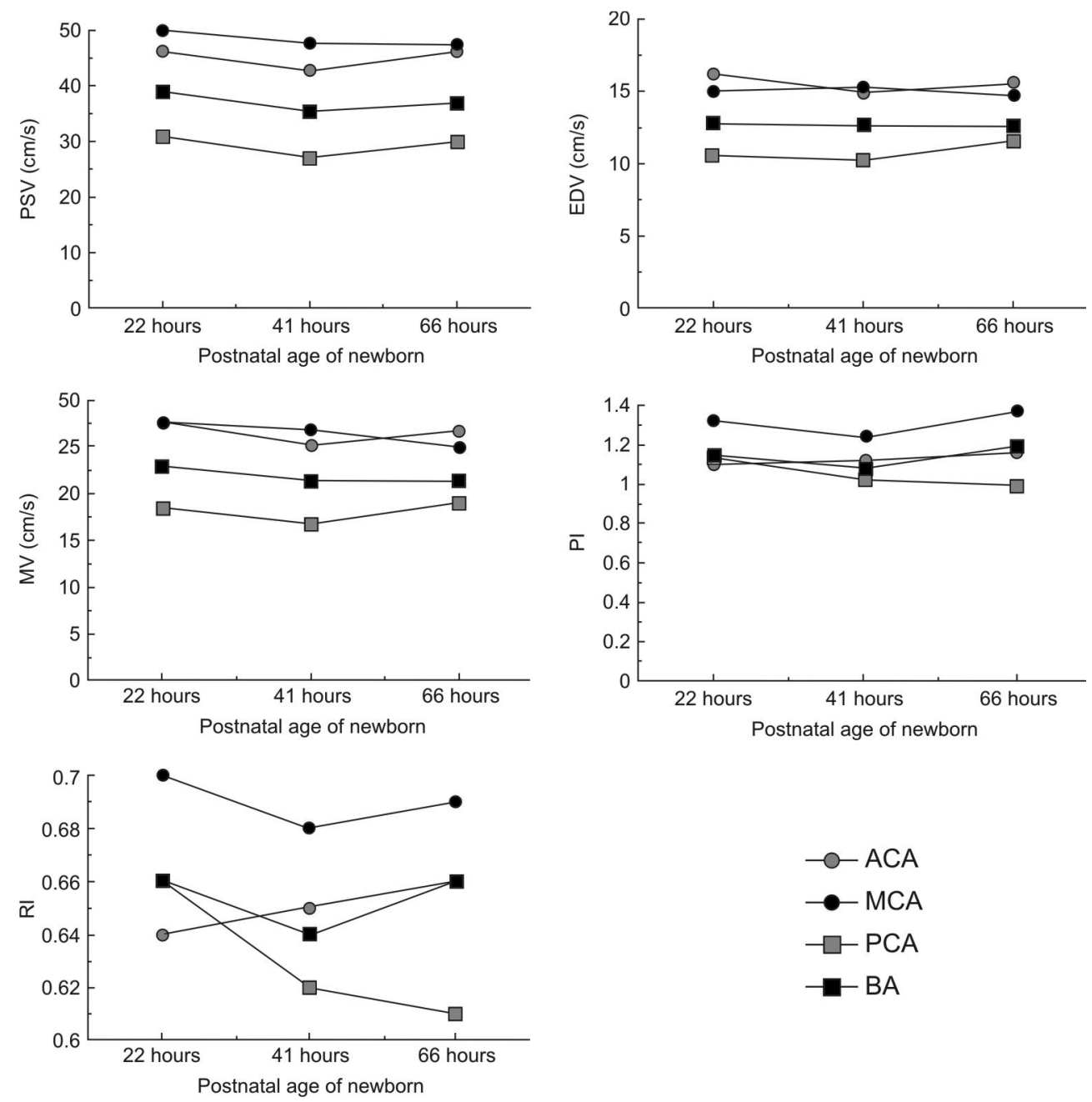

Fig 2. Graph of correlation between postnatal age of newborn and peak systolic velocity (PSV); MV: mean velocity; EDV: end diastolic velocity; PI: pulsatility index; RI: resitance index.

well with changes in CBF and allow a significant improvement in accuracy over instantaneous velocity or RI measurements isolated, and that RI correlated well with brain perfusion pressure but correlated weakly with cerebrovascular resistance and is not a good predictor of changes in cerebral blood flow ${ }^{15}$.

Seibert et al. ${ }^{16}$ studied the CBFV and RI in the anterior cerebral artery, middle cerebral artery and carotid artery in 57 healthy neonates and reported that average RI for healthy newborns was $0.75 \pm 0.10$ during the first $24 \mathrm{~h}$ of life, and that was a wide variation of normal and an overlap between normal and abnormal values.

In neonate with asphyxia RI is frequently low due to increase diastolic blood flow, but also can be higher if there is associated cerebral edema, hemorrhage, patent ductus arteriosus, cardiac ischemia due to decreased cerebral perfusing pressure rather than from increase of ce- rebral vascular impedance. There is a direct linear correlation between RI and increased of intracranial pressure particularly when associated a subdural efusion and hydrocephalus ${ }^{7,18}$.

Stark et al. ${ }^{19}$ were established initial RI values ranged $0.23-0.59$ in 16 neonates with history asphyxia in the first day of life. Archer et al. ${ }^{20}$ found no normal infant ever had a value of 0.55 or below during the first five days of life.

Recently, Swarup et al. ${ }^{21}$ described that blood flow velocity was lower and more variable in the left compared to the right middle cerebral artery on first day of life in premature infants, and speculate that this difference could be due to the associated ductus arteriosus patency. Pezzati et al. ${ }^{6}$ and Bokiniec et al. ${ }^{8}$, reported that there was no difference in CBFV in both brain hemispheres in healthy term neonates. Assis, et al. ${ }^{13}$ described no significant difference in RI between right and left ACA and MCA 
Table 5. Mean and medians of cerebral blood flow velocities $(\mathrm{cm} / \mathrm{s}), P I$ and RI in all cerebral arteries studied in relation to time after feeding of newborn. Kruskal-Kallis test ( $p>0.05$ ).

\begin{tabular}{|c|c|c|c|c|c|c|c|}
\hline \multicolumn{2}{|c|}{ Time after feeding (hour) } & \multirow{2}{*}{$\frac{n}{17}$} & \multirow{2}{*}{$\begin{array}{c}\text { ACA-PSV } \\
43.65\end{array}$} & \multirow{2}{*}{$\begin{array}{c}\text { ACA-EDV } \\
14.71\end{array}$} & \multirow{2}{*}{$\frac{\text { ACA-MV }}{25.65}$} & \multirow{2}{*}{$\begin{array}{c}\text { ACA-PI } \\
1.13\end{array}$} & \multirow{2}{*}{$\begin{array}{c}\text { ACA-RI } \\
0.66\end{array}$} \\
\hline Mean & $1 \mathrm{~h}$ & & & & & & \\
\hline Median & & & 43 & 16 & 25 & 1.06 & 0.65 \\
\hline Mean & $2 \mathrm{~h}$ & 24 & 44.13 & 15.04 & 25.71 & 1.14 & 0.66 \\
\hline Median & & & 45.5 & 15.5 & 26.5 & 1.14 & 0.66 \\
\hline Mean & $3 \mathrm{~h}$ & 9 & 46.56 & 17.67 & 28.22 & 1.05 & 0.62 \\
\hline Median & & & 47 & 16 & 27 & 0.97 & 0.63 \\
\hline \multicolumn{2}{|c|}{ Time after feeding (hour) } & $\mathrm{n}$ & RMCA-PSV & RMCA-EDV & RMCA-MV & RMCA-PI & RMCA-RI \\
\hline Mean & $1 \mathrm{~h}$ & 17 & 48.06 & 14.18 & 25.76 & 1.36 & 0.7 \\
\hline Median & & & 47 & 16 & 27 & 1.33 & 0.7 \\
\hline Mean & $2 \mathrm{~h}$ & 24 & 46.63 & 14.5 & 25.5 & 1.29 & 0.69 \\
\hline Median & & & 47.5 & 14 & 25 & 1.23 & 0.68 \\
\hline Mean & $3 \mathrm{~h}$ & 9 & 52.56 & 18.33 & 30.44 & 1.16 & 0.65 \\
\hline Median & & & 52 & 17 & 30 & 1.16 & 0.66 \\
\hline \multicolumn{2}{|c|}{ Time after feeding (hour) } & $\mathrm{n}$ & LMCA-PSV & LMCA-EDV & LMCA-MV & LMCA-PI & LMCA-RI \\
\hline Mean & $1 \mathrm{~h}$ & 17 & 49.76 & 15.41 & 27.35 & 1.27 & 0.69 \\
\hline Median & & & 49 & 14 & 27 & 1.3 & 0.69 \\
\hline Mean & $2 \mathrm{~h}$ & 24 & 45.63 & 14.42 & 25.08 & 1.26 & 0.68 \\
\hline Median & & & 44 & 15 & 25.5 & 1.27 & 0.67 \\
\hline Mean & $3 \mathrm{~h}$ & 9 & 49 & 16.56 & 28.11 & 1.19 & 0.66 \\
\hline Median & & & 51 & 14 & 28 & 1.18 & 0.67 \\
\hline \multicolumn{2}{|c|}{ Time after feeding (hour) } & $\mathrm{n}$ & RPCA-PSV & RPCA-EDV & RPCA-MV & RPCA-PI & RPCA-RI \\
\hline Mean & $1 \mathrm{~h}$ & 17 & 29.94 & 11.18 & 18.88 & 1 & 0.62 \\
\hline Median & & & 27 & 12 & 18 & 1 & 0.61 \\
\hline Mean & $2 \mathrm{~h}$ & 24 & 28 & 10 & 16.83 & 1.09 & 0.64 \\
\hline Median & & & 27 & 10 & 16.5 & 1.09 & 0.64 \\
\hline Mean & $3 \mathrm{~h}$ & 9 & 28.33 & 11.33 & 18 & 0.96 & 0.6 \\
\hline Median & & & 27 & 12 & 19 & 1 & 0.61 \\
\hline \multicolumn{2}{|c|}{ Time after feeding (hour) } & $\mathrm{n}$ & LPCA-PSV & LPCA-EDV & LPCA-MV & LPCA-PI & LPCA-RI \\
\hline Mean & $1 \mathrm{~h}$ & 17 & 29.65 & 11 & 18 & 1.04 & 0.62 \\
\hline Median & & & 30 & 10 & 17 & 1 & 0.63 \\
\hline Mean & $2 \mathrm{~h}$ & 24 & 26.17 & 9.71 & 15.79 & 1.04 & 0.62 \\
\hline Median & & & 27.5 & 10 & 16 & 1.06 & 0.63 \\
\hline Mean & $3 \mathrm{~h}$ & 9 & 30.22 & 11.44 & 18.56 & 1.01 & 0.62 \\
\hline Median & & & 31 & 11 & 18 & 1 & 0.62 \\
\hline \multicolumn{2}{|c|}{ Time after feeding (hour) } & $\mathrm{n}$ & BA-PSV & BA-EDV & BA-MV & BA-PI & BA-RI \\
\hline Mean & $1 \mathrm{~h}$ & 17 & 36 & 12.65 & 21.24 & 1.14 & 0.65 \\
\hline Median & & & 35 & 12 & 20 & 1 & 0.66 \\
\hline Mean & $2 \mathrm{~h}$ & 24 & 36.17 & 12.46 & 21.58 & 1.1 & 0.65 \\
\hline Median & & & 35 & 12 & 22 & 1.05 & 0.65 \\
\hline Mean & $3 \mathrm{~h}$ & 9 & 39 & 13.22 & 23.11 & 1.13 & 0.66 \\
\hline Median & & & 38 & 13 & 24 & 1.14 & 0.65 \\
\hline
\end{tabular}

RMCA: right middle cerebral artery; LMCA: left middle cerebral artery; ACA: anterior cerebral artery; BA: basilar artery; RPCA: right posterior cerebral artery; LPCA: left posterior cerebral artery; PSV: peak systolic velocity; EDV: end diastolic velocity; MV: mean velocity; PI: pulsatility index; RI: resistance index; $\mathrm{n}$ : number of cases.

in a group of 45 preterm healthy newborns. Our results demonstrated that the CBFV, PI and RI were significantly more higher in MCA than ACA, and more than PCA, showing regional difference of $C B F$, but there was no signifi- cant difference in CBFV, PI and RI between right and left MCA and between right and left PCA, demonstrating that there was no vascular dominant hemisphere in full term healthy newborns. 

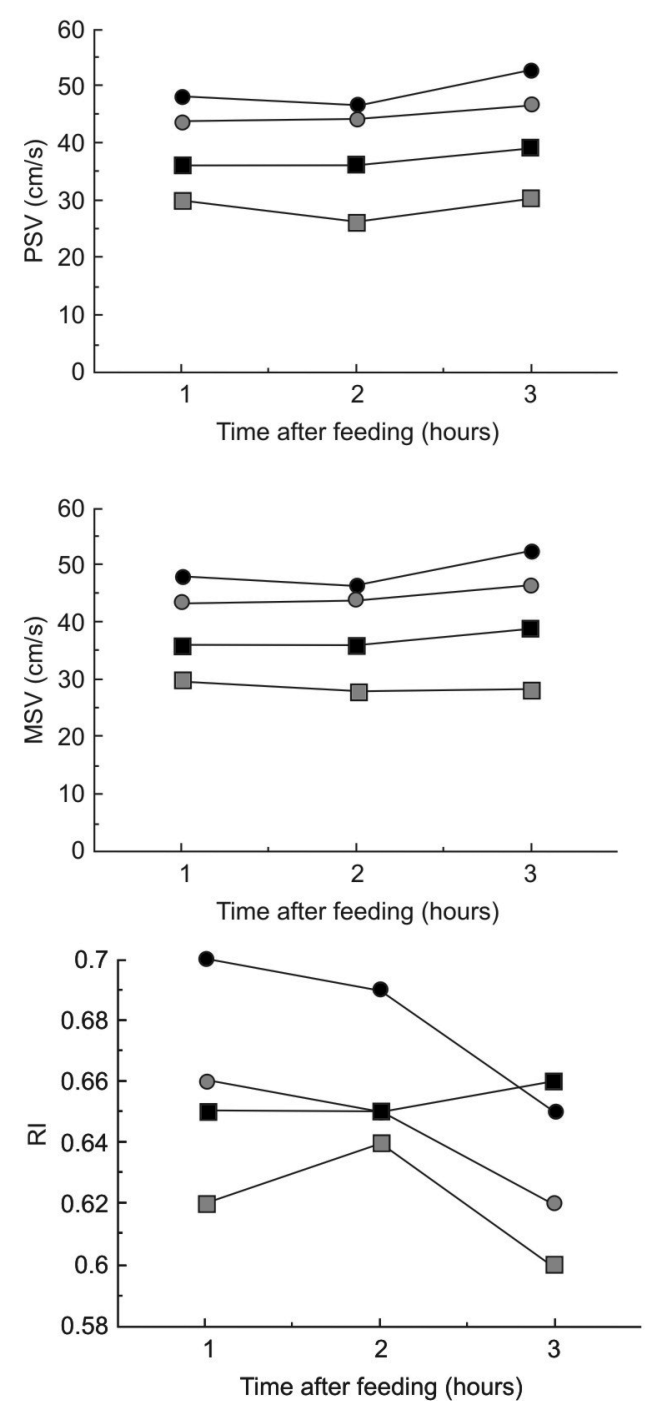
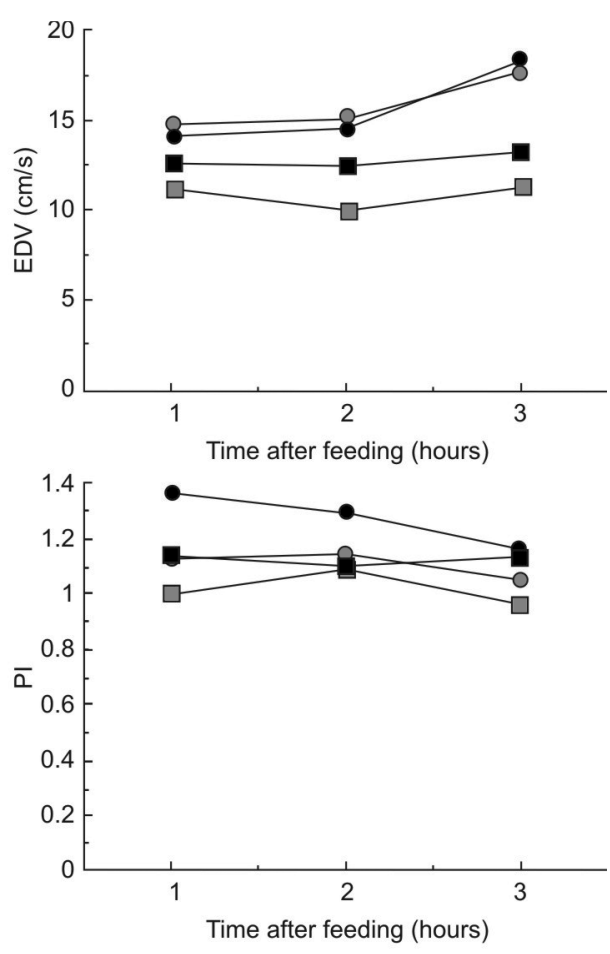

Fig 3. Graph of correlation between time after feeding of newborn and peak systolic velocity (PSV); MV: mean velocity; EDV: end diastolic velocity; PI: pulsatility index; RI: resitance index.

Baytur et al. ${ }^{22}$ studied 43 healthy term neonates who were delivered vaginally $(n=20)$ and by caesarean section ( $n=23)$, using Doppler ultrasound in middle cerebral artery and venous cerebral transverse sinus before delivery, and $1 \mathrm{~h}$ and $24 \mathrm{~h}$ after birth and observed that mode of delivery does not affect cerebral blood velocities. We observed similar findings in our study. The CBFV, PI and RI not changed significantly with the type of delivery.

Newborns males are more sensitive to brain injury and have higher morbidity and mortality than newborns females. Researches aiming at explaining the vulnerability in male infants has been intensified in the last years, and exposure to circulating sex steroids, that are potent hormones that exert a wide spectrum of influences on de- veloping fetal organs is felt to be a chief contributor to this phenomenon ${ }^{23}$.

Kehrer et al. ${ }^{24}$, studied quantitative measurement of cerebral blood flow volume performed by sonographic flowmetry of both internal carotid and vertebral arteries in healthy preterm and term infants in order to delineate the physiological characteristics of brain perfusion and they found no difference in cerebral blood flow volume between the sexes. In our study we demonstrated similar findings, the CBFV, PI and RI not changed significantly with the sex of newborn.

Usually, in term healthy newborns in the first days of life, the CBFV increase with increasing gestational age, postnatal age and birthweight, and RI increase with in- 
creasing gestational age. Studies has shown that there was no correlation between the mean blood pressure and mean blood flow velocity in cerebral arteries, and that this change is likely to represent a normal adaptive response of the cerebral circulation to postnatal life, when the efficiency of the CBF autoregulatory response in general improved with increasing age and maturity of the brain ${ }^{6,25}$. In the 50 full term healthy newborns studied between $12 \mathrm{~h}$ and $72 \mathrm{~h}$ of life, the results showed not statistically differences on CBFV, PI and RI.

In relation to influence of feeding on CBF, Nelle et al. ${ }^{26}$ using a transcranial Doppler ultrasound in preterm infants reported that bolus feeding provokes a considerable early decrease in CBFV in first 5 to 11 minutes after feeding with prefeeding values reached after 17 minutes.

Coombs ${ }^{27}$ and Badaró-Marques et al. ${ }^{28}$ reported that enteral feeding induces a significant and progressive increase in blood flow velocity in the superior mesenteric artery. Martinussen et al. ${ }^{29}$ studied healthy term neonates and described that postprandial increase in superior mesenteric artery mean velocity was not associated with changes in cardiac output and blood pressure; however, a fall in relative mesenteric vascular resistance suggesting regional redistribution of cardiac output, and that middle cerebral artery mean velocity increase was associated with an increase in blood pressure, and also observed that relative fraction of cardiac output to middle cerebral artery increased during the first days of life, suggesting a redistribution of blood flow to the metabolically active organs in the neonatal period.

Temporal changes in cerebral blood flow induced by feeding and postprandial period was investigated in our study by evaluation of newborns in $1 \mathrm{~h}, 2 \mathrm{~h}$ and $3 \mathrm{~h}$ after breastfeeding and the results demonstrated not statistically significant differences in the Doppler parameters. The oral feeding leads a vasodilation in the splanchnic area, with elevation of systolic velocity in the superior mesenteric artery and consequent increase of intestinal blood flow. After feeding to maintain constant perfusion of brain, small arteries and arterioles must vasoconstrict during increases in blood pressure to attenuate flow, and vasodilate during decreases in blood pressure, so producing a "autoregulatory plateu". This process occur specially in small resistance vessels, but may occur in vessels as proximal as the circle of Willis. The mechanisms by wich the process takes place may be intrinsic in the arteriolar wall, or be related to changes wich occur in relation to other stimuli such as arterial carbon dioxide tension $\left(\mathrm{PaCO}_{2}\right)$, oxygen concentration $\left(\mathrm{CO}_{2}\right)$, or glucose concentration levels, wich in turn lead to alterations in vascular tone to maintain cerebral blood flow ${ }^{30}$.

In conclusion, this cranial color Doppler ultrasonography study in full term healthy newborns with postnatal age between $12 \mathrm{~h}$ and $72 \mathrm{~h}$ of life demonstrated that there was not statistically significant differences on CBFV, PI and $\mathrm{RI}$ in relation to type of delivery, sex, postnatal age and time post feeding from $1 \mathrm{~h}$ to $3 \mathrm{~h}$. In this context, we believe that the knowledge of this cerebral blood flow profile in the first days of life can contribute to radiologists and pediatricians in an accurate interpretation of the cranial Doppler abnormal findings when pathologic flow velocities are analyzed.

\section{REFERENCES}

1. Lou HC, Lassen N, Friis-Hausen B. Impaired autoregulation of cerebral blood flow in the distressed newborn infant. J Pediatr 1979;94:118-121.

2. Bada H, Hajar W, Chua C, Sumner DS. Noninvasive diagnosis of neonatal asphyxia and intraventricular hemorrage by Doppler ultrasound. J Pediatrics 1979;95:775-779.

3. Horgan JG, Rumack CM, Hay T, Manco-Johnson MI, Merenstein GB, Esola C. Absolute intracranial blood-flow velocities evaluated by duplex Doppler sonography in asymptomatic preterm and term neonates. Ajr Am J Roentgenol 1989; 152:1059-1064.

4. Deeg KH, Rupprecht TR. Pulsed Doppler sonographic measurement of normal values for the flow velocity in the intracranial arteries of healthy newborns. Pediatr Radiol 1989;19:71-78.

5. Allison JW, Faddis LA, Kinder Dl, Roberson PK, Glasier CM, Seibert JJ. Intracranial resistive index (RI) values in normal term infants during the first day of life. Pediatr Radiol 2000;30: 618-620.

6. Pezzati M, Dani C, Biadaioli R, et al. Early postnatal Doppler assessment of cerebral blood flow velocity in healthy preterm and term infants. Dev Med Child Neurol 2002;44:745-752.

7. Muniz IACC, Netto AA, Gonçalves VMG. Velocimetria Doppler no período neonatal em recém-nascidos a termo pequenos para a idade gestacional. Arq Neuropsiquiatr 2003;61:808-815.

8. Bokiniec R, Kornacka MK, Czajkowski K. Pulsed Doppler velocimetry of cerebral and abdominal blood flow in infants of diabetic mothers J Ped Neonat 2006;31-35.

9. Pourcelot L. Diagnostic ultrasound for cerebral vascular diseases. In Donaldi J, Levis S (Eds). Present and future of diagnostic ultrasound. Rotterdam: Kooyter, 1976:141-147.

10. Gosling RG, King DH. Ultrasound angiology. In Marcus AW, Adamson J (Eds). Arteries and veins, $1^{\text {st }}$ Ed. Edinburg: Churchill-Livingstone 1975:61-71.

11. Lorek A, Takei Y, Cady EB, et al. Delayed ("secondary") cerebralenergy failure after acute hypoxia-ischemia in the newborn piglet: continuous 48-hours studies by phosporusmagnetic resonance spectroscopy. Pediatr Res 1994;36:699-706.

12. Eken P, Toet MC, Groendaal F, de Vries LS. Predictive value of early neuroimaging, pulsed Doppler and neurophysiology in ful term infants with hypoxic-ischaemic encephalopathy. Arch Dis Child 1995;73:75-80.

13. Assis MC, Machado, HR. Ecografia transfontanelar com fluxo a cores em recém-nascidos prematuros. Arq Neuropsiquiatr 2004;62:68-74. 
14. Hansen N, Stonestreet S, Rosenkrantz TS, Oh W. Validity of Doppler measurements of anterior cerebral artery blood flow velocity: Correlation with brain blood flow in piglets. Pediatrics 1983;72:526-531.

15. Taylor GA, Short Bl, Walker LK, Traysman RJ. Intracranial blood flow: quantification with duplex Doppler and color Doppler flow US. Radiology 1990;176:231-236.

16. Seibert, JJ, Mccowan TC, Chadduck WM, et al. Duplex pulsed Doppler us versus intracranial pressure in the neonate: clinical and experimental studies. Radiology 1989;171:155-159.

17. Goh D, Minns RA, Hendry GM, Thambyayah M, Steers AJ. Cerebrovascular resistive index assessed by duplex Doppler sonography and its relationship to intracranial pressure in infantile hydrocephalus. Pediatr Radiol 1992;22:246-250.

18. Taylor GA, Madsen JR. Neonatal hydrocephalus: hemodynamic response to fontanelle compression correlation with intracranial pressure and need for shunt placement. Pediatr Radiol 1996;201:685.

19. Stark JE, Seibert JJ. Cerebral artery Doppler ultrasonography for prediction of outcome after perinatal asphyxia. J Ultrasound Med 1994;13:595-600.

20. Archer LN, Leven MI, Evans DH. Cerebral artery Doppler ultrasonography for prediction of outcome after perinatal asphyxia. Lancet 1986;2:1116-1118.

21. Swarup J, Baker RW, Brozanski BW, Yanowitz TD. Asymmetry of cerebral blood flow velocity in low birth weight infants. Biol Neonate 2005;87:145-151.

22. Baytur, YB, Tarhan S, Uyar Y, Ozcakir HT, Lacin S, Coban
B, Inceboz U, Caglar H. Assessment of fetal cerebral arterial and venous blood flow before and after vaginal delivery or cesarean section. Ultrasound Obstetr Gynecol 2004;5: 522-528.

23. Renolleau S, Fau S, Charriaut-Marlangue C. Gender-related differences in apoptotic pathways after neonatal cerebral ischemia. Neuroscientist 2008;14:46-52.

24. Kehrer M, Krägeloh-Mann I, Goelz R, Schöning M. The development of cerebral perfusion in healthy preterm and term neonates. Neuropediatrics 2003;36:281-286.

25. Volpe JJ. Neurology of the newborn. 4th edition. Philadelphia, PA; WB Saunders, 2001.

26. Nelle M, Hoecker C, Linderkamp O. Effects of bolus tube feeding on cerebral blood flow velocity in neonates. Arch Dis Child Fetal Neonatal 1997;76:54-56.

27. Coombs RC, Morgan ME, Durbin GM, Booth IW, Mcneish AS. Doppler assessment of human neonatal gut blood flow velocities: postnatal adaptation and response to feeds. J Pediatr Gastroenterol Nutr 1992;15:6-12.

28. Badaró-Marques CS, Casanova, LD, Aranha, CA, Segre, CAM. Dopplervelocimetry of superior mesenteric artery in term newborns. Acta Cirurg Bras 2002;17:299-311.

29. Martinussen M, Brubakk AM, Linker DT, Vik T, Yao AC. Mesenteric blood flow velocity and its relation to circulatory adaptation during the first week of life in healthy term infants. Pediatr Res 1994;36:334-349.

30. Pryds O. Control of cerebral circulation in the high-risk Neonate. Ann Neurol 1991;30:321-329. 